\title{
A new facile synthesis of steroid dimers containing $17,17^{\prime}$-dicarboxamide spacers
}

\author{
Rui M. B. Carrilho ${ }^{\mathrm{a}, \mathrm{c}}$, Mariette M. Pereira ${ }^{\mathrm{a}, *}$, Maria José S. M. Moreno ${ }^{\mathrm{b}}$, Attila Takács ${ }^{\mathrm{c}, \mathrm{d}}$, László Kollár ${ }^{\mathrm{c}, \mathrm{d}, *}$ \\ a Departamento de Química, Universidade de Coimbra, Rua Larga, 3004-535 Coimbra, Portugal \\ ${ }^{\mathrm{b}}$ Faculdade de Farmácia, Universidade de Coimbra, Pólo das Ciências da Saúde, 3000-548 Coimbra, Portugal \\ c Department of Inorganic Chemistry, University of Pécs and János Szentágothai Science Center, PO Box 266, H-7624 Pécs, Hungary \\ ${ }^{\mathrm{d}}$ MTA-PTE Research Group for Selective Chemical Syntheses, Hungary
}

\section{A R T I C L E I N F O}

\section{Article history:}

Received 28 November 2012

Revised 4 February 2013

Accepted 12 February 2013

Available online 21 March 2013

\section{Keywords:}

Steroid dimers

Carbonylation

Palladium

Diamine

Carbon monoxide

\begin{abstract}
A B S T R A C T
A set of new steroid dimers linked through ring D-ring D were synthesized via catalytic diaminocarbonylation of 17 -iodo- $5 \alpha$-androst-16-ene, in the presence of palladium-phosphine in situ catalysts and aliphatic or aromatic diamines as $N$-nucleophiles. The dimeric steroidal compounds containing 17,17'dicarboxamide spacers were obtained through highly chemoselective reactions in good isolated yields and completely characterized by spectroscopic techniques.
\end{abstract}

(c) 2013 Elsevier Ltd. All rights reserved.
Steroids are widely found in both plant and animal kingdoms, and play crucial roles in biological systems. ${ }^{1,2}$ Among them, dimeric steroids constitute a class of compounds, which have recently attracted much attention due to their remarkable properties as potential cytotoxic, antimalarial, anticancer, and cholesterol lowering drugs as well as molecular umbrellas in drug delivery. ${ }^{3-9}$ In addition to their pharmacological importance, several dimeric and oligomeric steroids display micellar activity, ${ }^{10}$ they can also act as ligands for proteins and trigger cellular processes or may promote the affinity of ligands to their binding locations by providing extra anchoring points to the active site of certain domains. $^{11,12}$ Some of the dimers show liquid-crystal behaviour ${ }^{13}$ and play key roles in rate enhancement from hydrophobic binding. ${ }^{14}$

The synthetic approaches reported so far, have led to the preparation of cyclic and acyclic steroidal dimers, by connection between two cyclopentano-perhydrophenanthrene skeletons (through A-A, B-B, C-C, D-D or A-D rings). ${ }^{3-5}$ The steroidal moieties could be directly linked, ${ }^{15-17}$ linked through spacer groups ${ }^{18-25}$ and by connection through the steroidal side chains. ${ }^{26-29}$ Concerning steroidal D-D ring dimers linked through spacer groups, a number of pollutant multi-step and low-yielding synthetic approaches have

\footnotetext{
* Corresponding authors. Tel.: +351 239 854474; fax: +351 239827703 (M.M.P.). E-mail addresses: mmpereira@qui.uc.pt (M.M. Pereira), kollar@ttk.pte.hu (L. Kollár).
}

been frequently used. ${ }^{3}$ Thus, other environmentally sustainable alternatives to promote steroid dimerizations still constitute a great challenge. It is well established that transition-metal-catalyzed reactions are versatile tools to introduce different functionalities into specific positions of the steroidal framework, which can render marked changes in their biological properties. ${ }^{30}$ Recently, our groups have reported several examples on the aminocarbonylation of steroidal alkenyl-iodides toward carboxamides. ${ }^{31}$ Nevertheless, to the best of our knowledge, there is only one example on the synthesis of dimeric steroids involving Pd-catalyzed carbonylative dimerization of alkenyl-iodide intermediates, to form 17-carboxylic anhydrides, in the presence of carbon monoxide and water. ${ }^{32}$ It should be also noted that the application of diamines as $N$-nucleophiles in palladium-catalyzed aminocarbonylation toward dicarboxamides is unprecedented.

Therefore, the iodoalkene-based catalytic synthetic strategy described herein provides a completely new, facile, efficient, and atom economic methodology for the preparation of steroid dimers. A set of dimeric androst-16-enes with structurally different dicarboxamide spacers at C-17 was synthesized via one-pot, one-step palladium-catalyzed aminocarbonylation of 17 -iodo- $5 \alpha$-androst16-ene (1), which was obtained by the modified Barton's procedure from the corresponding 17-ketone. ${ }^{33}$

In a typical experiment, ${ }^{34} 17$-iodo-5 $\alpha$-androst-16-ene (1) was introduced in the autoclave in the presence of palladium(II) acetate, $2 \mathrm{M}$ equiv of triphenylphosphine, and $0.5 \mathrm{M}$ equiv of the desired diamine (1,2-diaminoethane (a), 1,4-diaminobutane (b), 


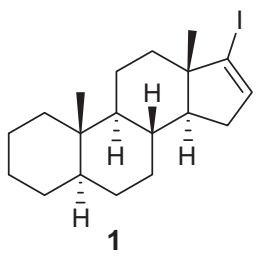

(
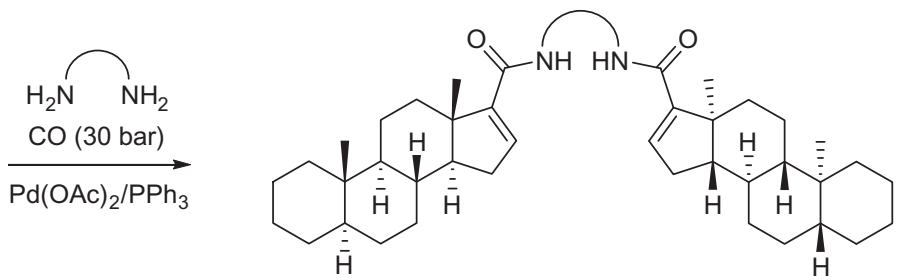

2 a : $91 \%$

2 b : $95 \%$

2 c : $48 \%$

2 d : $81 \%$

Scheme 1.

1,4-diaminobenzene (c), and (1S,2S)-(+)-1,2-diaminocyclohexane (d) as $N$-nucleophile), Scheme 1.

The carbonylative dimerization reaction leading to steroidal dicarboxamides (2a-2d) took place under relatively mild conditions ( 30 bar $\mathrm{CO}, 100^{\circ} \mathrm{C}$ ), with nearly full conversion of the initial iodo alkenyl steroid in $5 \mathrm{~h}$. The symmetric androst-16-ene dicarboxamide dimers $\mathbf{2 a}, \mathbf{2 b}, \mathbf{2 c}$, and $\mathbf{2 d}$ were obtained in $91 \%, 95 \%, 48 \%$, and $81 \%$ isolated yields, ${ }^{35}$ respectively, after work-up and purification by column chromatography using silica gel, or by simple washing with ethyl acetate. From these results, it was possible to conclude that aliphatic diamines (a), (b), and (d) gave significantly higher yields than that obtained with the aromatic diamine 1,4diaminobenzene $(\mathbf{c})$. It should be noted that the use of optically pure $(1 S, 2 S)-(+)$-diaminocyclohexane $(\mathbf{d})$ produced a single diastereomeric steroid dimer (2d) in $81 \%$ isolated yield. However, when a racemic mixture of trans-( \pm -1,2-diaminocyclohexane was used as nucleophile, a 1:1 mixture of two diastereomeric steroidal dimers was obtained, as evidenced by duplicate signals on ${ }^{1} \mathrm{H}$ and ${ }^{13} \mathrm{C}$ NMR spectra.

Detailed NMR and MS studies carried out on reaction mixtures and raw products (i.e., on isolated products prior to chromatography) revealed that no double carbon monoxide insertion into the Pd-alkenyl species occurred. That is, the formation of 2-ketocarboxamide-type derivatives could not be observed. This observation is in agreement with previous findings showing that iodoalkene substrates undergo double carbon monoxide insertion only under special conditions. ${ }^{36}$

In conclusion, a promising synthetic strategy was developed for the preparation of steroid dimers, via optimized palladium-catalyzed diaminocarbonylation reactions. The versatility of this methodology is demonstrated by the ability to prepare valuable functionalized steroid dimers linked through multiple achiral and a chiral dicarboxamide spacers.

\section{Acknowledgments}

The authors thank the financial support from Portuguese Fundação para a Ciência e a Tecnologia (FCT/QREN/FEDER/COMPETEPrograma Operacional Factores de Competitividade, PTDC/QUIQUI/112913/2009), the Hungarian Research Fund (CK78553) and
Developing Competitiveness of Universities in the Transdanubian Region (SROP-4.2.1.B-10/2/KONV-2010-0002 and SROP-4.2.2./B16 10/1-2010-0029). R.M.B.C. thanks FCT for a PhD grant (SFRH/ $\mathrm{BD} / 60499 / 2009)$. The help of T. Loránd in the IR analysis is kindly acknowledged.

\section{References and notes}

1. Maltais, R.; Poirier, D. Steroids 2011, 76, 929-948

2. Ross, D. A.; Cetas, J. S. J. Neurooncol. 2012, 109, 439-447.

3. Nahar, L.; Sarker, S. D. Steroid Dimers Chemistry and Applications in Drug Delivery; John Wiley \& Sons: Chichester, 2012. pp 67-89.

4. Li, Y.; Dias, J. R. Chem. Rev. 1997, 97, 283-304.

5. Nahar, L.; Sarker, S. D.; Turner, A. B. Curr. Med. Chem. 2007, 14, 1349-1370.

6. Moser, B. R. J. Nat. Prod. 2008, 71, 487-491.

7. López-Antón, N.; Rudy, A.; Barth, N.; Schmitz, L. M.; Pettit, G. R.; SchulzeOsthoff, K.; Dirsch, V. M.; Vollmar, A. M. J. Biol. Chem. 2006, 281, 33078-33086.

8. Taber, D. F.; Joerger, J.-M. J. Org. Chem. 2008, 73, 4155-4159.

9. Phillips, S. T.; Shair, M. D. J. Am. Chem. Soc. 2007, 129, 6589-6598.

10. McKenna, J.; McKenna, J. M.; Thornthwaite, D. W. J. Chem. Soc., Chem. Commun. 1977, 809-811.

11. Clemons, P. A. Curr. Opin. Chem. Biol. 1999, 3, 112-115.

12. Diver, S. T.; Schreiber, S. L. J. Am. Chem. Soc. 1997, 119, 5106-5109.

13. Thiemann, T.; Vill, V. J. Phys. Chem. Ref. Data 1997, 26, 291-333.

14. Guthrie, J. P.; Cossar, J.; Dawson, B. A. Can. J. Chem. 1986, 64, 2456-2469.

15. Fournier, D.; Poirier, D. Bioorg. Med. Chem. Lett. 2009, 19, 693-696.

16. Shamsuzzaman; Alam, M. G.; Siddiqui, T. J. Chem. Sci. 2011, 123, 491-495.

17. Templeton, J. F.; Majgier-Baranowska, H.; Marat, K. Steroids 2000, 65, 219-223.

18. Morzycki, J. W.; Kalinowski, S.; Łotowski, Z.; Rabiczko, J. Tetrahedron 1997, 53 , 10579-10590.

19. Joachimiak, R.; Paryzek, Z. J. Incl. Phenom. Macrocycl. Chem. 2004, 49, 127-132.

20. Paryzek, Z.; Piasecka, M.; Joachimiak, R.; Luks, E.; Radecka-Paryzek, W. J. Rare Earths 2010, 28, 56-60.

21. Joachimiak, R.; Piasecka, M.; Paryzek, Z. J. Chem. Res. 2008, 5, 260-265.

22. Valverde, L. F.; Cedillo, F. D.; Tolosa, L.; Maldonado, G.; Reyes, G. C. J. Mex. Chem. Soc. 2006, 50, 42-45.

23. De Riccardis, F.; Di Filippo, M.; Garrisi, D.; Izzo, I.; Mancin, F.; Pasquato, L.; Scrimin, P.; Tecilla, P. Chem. Commun. 2002, 3066-3067.

24. Yus, M.; Soler, T.; Foubelo, F. Tetrahedron: Asymmetry 2001, 12, 801-810.

25. Sierra, M. A.; Torres, M. R.; de la Torre, M. C.; Álvaro, E. J. Org. Chem. 2007, 72, 4213-4219.

26. Rega, M.; Jiménez, C.; Rodríguez, J. Steroids 2007, 72, 729-735.

27. D’Auria, M. V.; Giannini, C.; Zampella, A.; Minale, L.; Debitus, C.; Roussakis, C. J. Org. Chem. 1998, 63, 7382-7388.

28. Edelsztein, V. C.; Di Chenna, P. H.; Burton, G. Tetrahedron 2009, 65, 3615-3623. 29. Nahar, L.; Turner, A. B. Steroids 2003, 68, 1157-1161.

30. Skoda-Földes, R.; Kollár, L. Chem. Rev. 2003, 103, 4095-4129.

31. (a) Ács, P.; Takács, A.; Kiss, M.; Pálinkás, N.; Mahó, S.; Kollár, L. Steroids 2011 76, 280-290; (b) Takács, A.; Ács, P.; Berente, Z.; Wölfling, J.; Schneider, G.; Kollár, L. Steroids 2010, 75, 1075-1081; (c) Ács, P.; Müller, E.; Czira, G.; Mahó S.; Pereira, M.; Kollár, L. Steroids 2006, 71, 875-879. 
32. Skoda-Földes, R.; Csákai, Z.; Kollár, L.; Szalontai, G.; Horváth, J.; Tuba, Z. Steroids 1995, 60, 786-790.

33. (a) Barton, D. H. R.; O’Brien, R. E.; Sternhell, S. J. Chem. Soc. 1962, 470-476; (b) Barton, D. H. R.; Bashiardes, B.; Fourrey, J.-L. Tetrahedron Lett. 1983, 24, 16051608.

34. In a typical experiment, $\mathrm{Pd}(\mathrm{OAc})_{2}(2.6 \mathrm{mg}, 0.0125 \mathrm{mmol})$, triphenylphosphine ( $6.6 \mathrm{mg}, 0.025 \mathrm{mmol}), 17$-iodo-androst-16-ene $(220 \mathrm{mg}, 0.57 \mathrm{mmol})$, diamine nucleophile $(0.285 \mathrm{mmol})$, and triethylamine $(0.25 \mathrm{ml})$ were dissolved in DMF $(5 \mathrm{~mL})$ under argon in a stainless steel autoclave. The atmosphere was pressurized to 30 bar CO. The reaction was conducted for $5 \mathrm{~h}$, upon stirring at $100^{\circ} \mathrm{C}$. The mixture was then evaporated to dryness; the residue was dissolved in chloroform $(20 \mathrm{ml})$ and washed with water $(3 \times 20 \mathrm{~mL})$. The organic phase was dried over $\mathrm{Na}_{2} \mathrm{SO}_{4}$, filtered, and evaporated to a crystalline material or to a waxy residue, which was analyzed by ${ }^{1} \mathrm{H}$ and ${ }^{13} \mathrm{C}$ NMR. All compounds were purified through column chromatography (Silicagel 60, Merck, $0.063-0.200 \mathrm{~mm}$ ), EtOAc/ $\mathrm{CHCl}_{3}$ (exact ratios are specified for each compound), or simply washed with ethyl acetate (as in the case of $\mathbf{2 c}$ ).

35. Characterization of the compounds 2 a-2d: $N, N^{\prime}$-(ethane-1,2-diyl)diandrost16-ene-17-carboxamide (2a): Yield: $163 \mathrm{mg}$ (91\%), white solid, mp 153$156{ }^{\circ} \mathrm{C} ; R_{\mathrm{f}}\left(\mathrm{EtOAc} / \mathrm{CHCl}_{3} 2: 1\right) 0.16 ; \delta_{\mathrm{H}}\left(400 \mathrm{MHz}, \mathrm{CDCl}_{3}\right) 6.35(2 \mathrm{H}, \mathrm{br} \mathrm{s}, \mathrm{NH}), 6.23$ $(2 \mathrm{H}, \mathrm{s}, \mathrm{C}=\mathrm{CH}), 3.45\left(2 \mathrm{H}, \mathrm{d}, 9.6 \mathrm{~Hz}, \mathrm{~N}-\mathrm{CH}_{\mathrm{a}} \mathrm{H}_{\mathrm{b}}\right), 3.33\left(2 \mathrm{H}, \mathrm{d}, 9.6 \mathrm{~Hz}, \mathrm{~N}-\mathrm{CH}_{\mathrm{a}} \mathrm{H}_{\mathrm{b}}\right)$, $2.03-2.12\left(4 \mathrm{H}, \mathrm{m}, 15-\mathrm{H}_{2}\right), 1.86(2 \mathrm{H}, \mathrm{dd}, 12.0 \mathrm{~Hz}, 15.2 \mathrm{~Hz}, 14-\mathrm{H}), 0.88(6 \mathrm{H}, \mathrm{s}, 19-$ $\left.\mathrm{H}_{3}\right), 0.74\left(6 \mathrm{H}, \mathrm{s}, 18-\mathrm{H}_{3}\right), 0.65-1.60(38 \mathrm{H}, \mathrm{m}$, skeleton protons $) ; \delta_{\mathrm{C}}(100.6 \mathrm{MHz}$, $\left.\mathrm{CDCl}_{3}\right) 167.4,150.4,136.4,56.9,55.4,47.3,46.7,39.8,38.7,36.6,35.0$, 33.9, 32.1, 31.8, 29.1, 29.0, 26.9, 22.3, 20.8, 16.6, 12.3; $\operatorname{IR~}\left(\operatorname{KBr}\left(\mathrm{cm}^{-1}\right)\right): 3295(\mathrm{v} \mathrm{br}$, $\mathrm{NH}$ ), 1645 (CO), $1591(\mathrm{C}=\mathrm{C})$; HRMS (ESI): $m / z$ calcd for $\mathrm{C}_{42} \mathrm{H}_{65} \mathrm{~N}_{2} \mathrm{O}_{2}[\mathrm{M}+\mathrm{H}]^{+}$ 629.5041 , found 629.5038. $N, N^{\prime}$-(Butane-1,4-diyl)diandrost-16-ene-17carboxamide (2b): Yield: $178 \mathrm{mg}(95 \%)$, white solid, $\mathrm{mp} 179-182{ }^{\circ} \mathrm{C} ; R_{\mathrm{f}}$
$\left(\mathrm{EtOAc} / \mathrm{CHCl}_{3} 1: 1\right) 0.15 ; \delta_{\mathrm{H}}\left(400 \mathrm{MHz}, \mathrm{CDCl}_{3}\right) 6.25(2 \mathrm{H}, \mathrm{s}, \mathrm{C}=\mathrm{CH}), 5.80(2 \mathrm{H}, \mathrm{br}$ s, $\mathrm{NH}), 3.26\left(4 \mathrm{H}\right.$, br s, $\left.\mathrm{NCH}_{2} \mathrm{CH}_{2} \mathrm{CH}_{2} \mathrm{CH}_{2} \mathrm{~N}\right), 2.02-2.15\left(4 \mathrm{H}, \mathrm{m}, 15-\mathrm{H}_{2}\right), 1.88(2 \mathrm{H}$, dd, $11.6 \mathrm{~Hz}, 15.2 \mathrm{~Hz}, 14-\mathrm{H}), 0.90\left(6 \mathrm{H}, \mathrm{s}, 19-\mathrm{H}_{3}\right), 0.75\left(6 \mathrm{H}, \mathrm{s}, 18-\mathrm{H}_{3}\right), 0.66-1.60$ $\left(42 \mathrm{H}, \mathrm{m}\right.$, skeleton protons $\left.+\mathrm{NCH}_{2} \mathrm{CH}_{2} \mathrm{CH}_{2} \mathrm{CH}_{2} \mathrm{~N}\right) ; \delta_{\mathrm{C}}\left(100.6 \mathrm{MHz}, \mathrm{CDCl}_{3}\right) 166.5$, $150.8,135.8,57.0,55.3,47.4,46.8,38.7$ (double intensity), 36.6, 35.2, 34.0, $32.1,31.8,29.2,29.1,27.2,27.0,22.3,20.9,16.7,12.3 ; \operatorname{IR}\left(\mathrm{KBr}\left(\mathrm{cm}^{-1}\right)\right): 3298(\mathrm{v}$ br, NH), 1639 (CO), $1594(\mathrm{C}=\mathrm{C})$; HRMS (ESI): $\mathrm{m} / \mathrm{z}$ calcd for $\mathrm{C}_{44} \mathrm{H}_{69} \mathrm{~N}_{2} \mathrm{O}_{2}[\mathrm{M}+\mathrm{H}]^{+}$ 657.5354 , found 657.5347. $N, N^{\prime}$-(1,4-phenylene)diandrost-16-ene-17carboxamide (2c): Yield: $92 \mathrm{mg}(48 \%)$, beige solid, $\mathrm{mp} 316-319{ }^{\circ} \mathrm{C}$ (dec.); $R_{\mathrm{f}}$ $\left(\mathrm{CHCl}_{3} /\right.$ EtOAc $\left.10: 1\right) 0.45 ; \delta_{\mathrm{H}}\left(400 \mathrm{MHz}, \mathrm{CDCl}_{3}\right) 7.50(4 \mathrm{H}, \mathrm{s}$, aromatic $H) 7.40$ $(2 \mathrm{H}, \mathrm{br} \mathrm{s}, \mathrm{NH}), 6.44(2 \mathrm{H}, \mathrm{s}, \mathrm{C}=\mathrm{CH}), 2.19-2.28\left(4 \mathrm{H}, \mathrm{m}, 15-\mathrm{H}_{2}\right), 2.02(2 \mathrm{H}$, dd, $9.4 \mathrm{~Hz}, 12.2 \mathrm{~Hz}, 14-\mathrm{H}), 1.04\left(6 \mathrm{H}, \mathrm{s}, 19-\mathrm{H}_{3}\right), 0.82\left(6 \mathrm{H}, \mathrm{s}, 18-\mathrm{H}_{3}\right), 0.76-1.70(38 \mathrm{H}$, $\mathrm{m}$, skeleton protons); $\delta_{\mathrm{C}}\left(100.6 \mathrm{MHz}, \mathrm{CDCl}_{3}\right) 164.1,151.3,136.8,134.3,120.4$, 56.9, 55.3, 47.4, 47.0, 38.6, 36.6, 35.1, 34.0, 32.1, 32.0, 29.2, 29.0, 26.9, 22.3, 20.8, 16.7, 12.3; IR ( $\left.\mathrm{KBr}\left(\mathrm{cm}^{-1}\right)\right): 3314(\mathrm{v}$ br, NH), $1665(\mathrm{CO}), 1594(\mathrm{C}=\mathrm{C})$; HRMS (ESI): $m / z$ calcd for $\mathrm{C}_{46} \mathrm{H}_{65} \mathrm{~N}_{2} \mathrm{O}_{2}[\mathrm{M}+\mathrm{H}]^{+}$677.5041, found 677.5032. $N, N^{\prime}-$ (Cyclohexane-(1S,2S)-diyl)diandrost-16-ene-17-carboxamide (2d): Yield: $157 \mathrm{mg}(81 \%)$, white solid, mp 230-233 ${ }^{\circ} \mathrm{C} ;[\alpha]_{\mathrm{D}}^{20}:+40.0\left(\mathrm{c} 1.0, \mathrm{CH}_{2} \mathrm{Cl}_{2}\right) ; R_{\mathrm{f}}$ $\left(\mathrm{CHCl}_{3} /\right.$ EtOAc $\left.8: 1\right) 0.8 ; \delta_{\mathrm{H}}\left(400 \mathrm{MHz}, \mathrm{CDCl}_{3}\right) 6.23(2 \mathrm{H}$, br s, $\mathrm{NH}), 6.20(2 \mathrm{H}, \mathrm{s}$, $\mathrm{C}=\mathrm{CH}), 3.72\left(2 \mathrm{H}, \mathrm{br} \mathrm{s}, \mathrm{N}-\mathrm{CH}_{2}\right), 2.03-2.16\left(6 \mathrm{H}, \mathrm{m}, 15-\mathrm{H}_{2}+\mathrm{NCHCH}_{\mathrm{a}} \mathrm{H}_{\mathrm{b}}, 1.93(2 \mathrm{H}\right.$, dd, $12.3 \mathrm{~Hz}, 15.6 \mathrm{~Hz}, 14-\mathrm{H}), 0.95\left(6 \mathrm{H}, \mathrm{s}, 19-\mathrm{H}_{3}\right), 0.80\left(6 \mathrm{H}, \mathrm{s}, 18-H_{3}\right), 0.68-1.80$ $\left(44 \mathrm{H}, \mathrm{m}\right.$, skeleton protons $\left.+\mathrm{NCHCH}_{\mathrm{a}} \mathrm{H}_{\mathrm{b}} \mathrm{CH}_{2} \mathrm{CH}_{2} \mathrm{CH}_{\mathrm{a}} \mathrm{H}_{\mathrm{b}} \mathrm{CHN}\right) ; \delta_{\mathrm{C}}(100.6 \mathrm{MHz}$, $\left.\mathrm{CDCl}_{3}\right) 167.1 ; 150.8,135.2,56.8,55.7,53.6,47.3,46.9,39.0,36.7,35.0,33.9$ 32.7, 32.1, 31.8, 29.2, 29.0 26.9, 25.0, 22.3, 20.8, 16.5, 12.3; $\operatorname{IR~}\left(\operatorname{KBr}\left(\mathrm{cm}^{-1}\right)\right)$ : $3340(\mathrm{v}$ br, $\mathrm{NH}), 1642(\mathrm{CO}), 1591(\mathrm{C}=\mathrm{C})$; HRMS (ESI): $m / z$ calcd for $\mathrm{C}_{46} \mathrm{H}_{71} \mathrm{~N}_{2} \mathrm{O}_{2}$ $[\mathrm{M}+\mathrm{H}]^{+}$683.5510, found 683.5528 .

36. Carrilho, R. M. B.; Pereira, M. M.; Takács, A.; Kollár, L. Tetrahedron 2012, 68, 204-207. 